\title{
A NOTE ON WEAKLY O-CONTINUOUS FUNCTIONS
}

\section{Mršević}

Department of Mathematics

Faculty of Sciences

University of Belgrade

Yugoslavia

\section{and I.L. Reilly}

Department of Mathematics

University of California, Davis Davis, California 95616

U.S.A.

(Received June 8, 1987 and in revised form February 9, 1988)

ABSTRACT. Recently a new class of functions between topological spaces, called weakly $\theta$-continuous functions, has been introduced and studied. In this paper we show how an appropriate change of topology on the domain of a weakly $\theta$-continuous function reduces it to a weakly continuous function. This paper examines some of the consequences of this result.

KEY WORDS AND PHRASES. Weak continuity, weakly $\theta$-continuous, change of topology . 1980 MATHEMATICS SUBJECT CLASSIFICATION. 54C10, 54 A10.

PRELIMINARIES.

Throughout this paper $X$ and $Y$ denote topological spaces on which no separation axioms are assumed unless explicitly stated. A subset $S$ of a space $X$ is said to be regular open (resp. regular closed) if int(c1S) $=\mathrm{S}$ (resp. cl(intS) = S), where c1S and ints denote the closure of $S$ and the interior of $S$, respectively. Henceforth we denote int(c1S) by the Bourbaki notation $\alpha(S)$. A point $x \in X$ is called a $\delta$-cluster (resp. $\theta$-cluster) point of $S$ if $S \cap \alpha(\mathrm{U}) \neq \phi$ (resp. $S \cap c 1(U) \neq \phi)$ for each open set $U$ containing $x$. The set of all $\delta$-cluster (resp. $\theta$-cluster) points of $\mathrm{S}$ is called the $\delta$-closure (resp. $\theta$-closure) of $S$ and is denoted by $c 1_{\delta}(S)$ (resp. $c 1_{\theta}(S)$ ). A subset $S$ is said to be $\delta$-closed (resp. $\theta$-closed) if $\mathrm{c} 1_{\delta}(\mathrm{S})=\mathrm{S}$ (resp. $\left.\mathrm{c} 1_{\theta}(\mathrm{S})=\mathrm{S}\right)$.

A subset is called $\delta$-open (resp. $\theta$-open) if its complement is $\delta$-closed (resp. $\theta$-closed). The family of all $\delta$-open (resp. $\theta$-open) subsets of $(X, T)$ forms a topology $T_{\delta}$ (resp. $T_{\theta}$ ) on $X$.

Since the intersection of two regular open subsets of $(X, T)$ is regular open, the family of all regular open subsets of $(X, T)$ forms a base for a topology $T_{s}$ on $X$, 
called the semi-regularization of $T$. It turns out that $T_{S}=T_{\delta}$, and that $T_{\theta} \subset T_{S} \subset T$ for any topological space $(X, T)$. The relationship between $T$ and $T_{s}$ is considered in [MRV]. In particular, we note that $(X, T)$ is semi-regular if and only if $T=T_{S}$.

A function $f: X \rightarrow Y$ is called weakly continuous (resp. $\theta$-continuous) if for each point $x \in X$ and each open neighbourhood $V$ of $f(x)$, there is an open neighbourhood $U$ of $x$ such that $f(U) \subset c 1 V$ (resp. $f(c 1 U) \subset c 1 V$ ). These concepts were considered by Levine [L] and Fomin [F] respectively.

CHANGE OF TOPOLOGY

DEFINITION 1. A function $\mathrm{f}:(\mathrm{X}, T) \rightarrow(\mathrm{Y}, U)$ is called weakly $\theta$-continuous if for each point $x \in X$ and each open neighbourhood $V$ of $f(x)$ there is an open neighbourhood $U$ of $x$ such that $f(\alpha(U)) \subset$ c1V .

Cammaroto and Noiri [CN] point out with their Examples 3.2 and 3.3 that the class of weakly $\theta$-continuous functions lies strictly between the class of $\theta$-continuous functions and the class of weakly continuous functions. In a recent paper [GMRV], Gauld, Vamanamurthy and the present authors observed that there are many properties closely related to the notion of continuity which in fact coincide with continuity if the topology on either the domain or the range or both is changed. They called such a property a continuity property of functions.

PROPOSITION 1. Weak $\theta$-continuity is not a continuity property.

PROOF. We refer to Lemma 2 of [GMRV]. It is shown in the proof of Theorem 2 of [GMRV] that $f$ and $g$ are $\theta$-continuous, and hence they are weakly $\theta$-continuous, while $h$ is not weakly continuous and hence not weakly $\theta$-continuous. So the result follows from Lemma 2 of [GMRV].

We now show that a change of topology is germane to the discussion of weak $\theta$-continuity. We observe that if the domain of a weakly $\theta$-continuous function is retopologized appropriately, the function reduces to a weakly continuous function. Baker [B2] has also made this observation. He used the term weakly $\delta$-continuous in place of weakly $\theta$-continuous. The point should be made that weak continuity is not a continuity property [GMRV, Theorem 2]. Recently, Noiri [N2] has studied another class of functions, the weakly $\alpha$-continuous functions, which also reduce to weakly continuous functions when the domain is retopologized appropriately [N2, Lemma 2.2] . The proof of Theorem 1 follows directly from Definition 1 . 
THEOREM 1. A function $f:(X, T) \rightarrow(Y, U)$ is weakly $\theta$-continuous if and only if $\quad \mathrm{f}:\left(\mathrm{X}, T_{\mathrm{S}}\right) \rightarrow(\mathrm{Y}, U)$ is weakly continuous.

Now most of the results of Camnaroto and Noiri [CN] can be reinterpreted in the light of Theorem 1. For example, their Theorem 3.4 is an immediate consequence of Theorem 1, their Theorem 3.5 (f) is a restatement of Theorem 1 of Levine [L], while Theorem 3.5(e) follows from Theorem 1(e) of Espelie and Joseph [EJ]. Other parts of Theorem 1 of [EJ] enable us to provide some further alternative characterizations of weak $\theta$-continuity.

PROPOSITION 2. For a function $f:(X, T) \rightarrow Y$ the following are equivalent:

(a) $f$ is weakly $\theta$-continuous.

(b) For each $B \subset Y, T_{s} c 1\left(f^{-1}\left(\operatorname{int}\left(c 1_{\theta} B\right)\right)\right) \subset f^{-1}\left(c 1_{\theta} B\right)$.

(c) For each open set $V$ in $Y, T_{s} c 1\left(f^{-1}(\alpha(V))\right) \subset f^{-1}(c 1 V)$.

(d) For each regularly closed subset $B$ of $Y, T_{s} c 1\left(f^{-1}(\right.$ int B $\left.)\right) \subset f^{-1}$ (B).

On the other hand, we can go in the opposite direction and use Theorem 3.7 of

[CN] to obtain the following result for weak continuity.

PROPOSITION 3 . If $f: X \rightarrow Y$ is weakly continuous then

(a) $f^{-1}(F)$ is closed for each $\theta$-closed subset $F$ of $Y$,

(b) $f^{-1}(V)$ is open for each $\theta$-open subset $V$ of $Y$.

The Example 3.9 of $[\mathrm{CN}]$ can be modified to show that the converse of Proposition 3 is false.

We observe that weak continuity is preserved under restriction of domain and under products. This observation together with Theorem 1 can be used to obtain the results of $[\mathrm{CN}, \S 4]$. For example, Lemma 4 of [MRV] gives Theorem 4.1 of [CN], while Lemma 3 of [MRV] provides a proof of Theorem 4.2 and 4.3 of [CN].

DEFINITION 2. A function $f:(X, T) \rightarrow(Y, U)$ is called

(i) almost open if $\mathrm{f}:\left(\mathrm{X}, \mathrm{T}_{\mathrm{S}}\right) \rightarrow(\mathrm{Y}, U)$ is open,

(ii) $\delta$-open if $f:\left(X, T_{s}\right) \rightarrow\left(Y, U_{s}\right)$ is open,

(iii)super open if $\mathrm{f}:(\mathrm{X}, T) \rightarrow\left(\mathrm{Y}, \mathrm{U}_{\mathrm{s}}\right)$ is open.

DEFINITION 3. A subset $\mathrm{S}$ of $(X, T)$ is called $N$-closed if for any open cover $C$ of $S$ there is a finite subcollection $D$ of $C$ such that $S \subset U\{\alpha(V): V \in D\}$. Applying this change of topology technique to Lemma 2 of Rose [R] gives the following result.

PROPOSITION 4 . Let $\mathrm{f}: \mathrm{X} \rightarrow \mathrm{Y}$ and $\mathrm{g}: \mathrm{Y} \rightarrow \mathrm{Z}$.

(a) If $g \circ f$ is weakly $\theta$-continuous and $f$ is an almost open surjection, then $g$ is weakly continuous. 
(b) If $g \circ f$ is weakly $\theta$-continuous and $f$ is an $\delta$-open surjection, then $g$ is weakly $\theta$-continuous.

(c) If $g \circ f$ is weakly continuous and $f$ is a super open surjection, then $g$ is weakly $\theta$-continuous.

PROPOSITION 5. If $f: X \rightarrow Y$ is weakly $\theta$-continuous and $Y$ is Hausdorff, then for each N-closed subset $C$ of $X, f(C)$ is $\delta$-closed in $Y$.

PROOF. Since $f:\left(X, T_{s}\right) \rightarrow(Y, U)$ is weakly continuous and $U_{s} \subset U$, we have $f:\left(X, T_{S}\right) \rightarrow\left(Y, U_{S}\right)$ is weakly continuous and hence subweakly continuous. If $C$ is $\mathrm{N}$-closed in $(X, T)$ then $\mathrm{C}$ is compact in $\left(X, T_{S}\right)$ so by Corollary 3 to Theorem 2 of Baker $[B 1], f(C)$ is closed in $\left(Y, U_{S}\right)$ and hence $\delta$-closed in $(Y, U)$.

Using a similar argument to that in the proof of Proposition 5, and Corollary 2 to Theorem 2 of Baker [B1], we obtain the following result.

PROPOSITION 6. If $\mathrm{f}: \mathrm{X} \rightarrow \mathrm{Y}$ is weakly $\theta$-continuous and $Y$ is Hausdorff, then for each $N$-closed subset $C$ of $Y, f^{-1}(C)$ is $\delta$-closed in $X$.

PROPOSITION 7. Let $Y$ be a Hausdorff space, $f: X \rightarrow Y$ be $\delta$-continuous and $g: X \rightarrow Y$ be weakly $\theta$-continuous. Then the set $\{x \in X: f(x)=g(x)\}$ is a $\delta$-closed subset of $X$.

PROOF. We have $f:\left(X, T_{S}\right) \rightarrow\left(Y, U_{S}\right)$ is continuous, $g:\left(X, T_{S}\right) \rightarrow\left(Y, U_{S}\right)$ is weakly continuous and $\left(Y, U_{S}\right)$ is Hausdorff, and apply Theorem 6 of Baker [B1].

Finally we observe that Theorem 7.8 of $[\mathrm{CN}]$ is a corollary of Theorem 10 of [N1]. For, if $f:(X, T) \rightarrow(Y, U)$ is weakly $\theta$-continuous then $f:(X, T) \rightarrow\left(Y, U_{S}\right)$ is weakly $\theta$-continuous, so that $f:\left(X, T_{s}\right) \rightarrow\left(Y, U_{s}\right)$ is weakly continuous. Hence $G(f)$ is closed in $\left(X \times Y, T_{S} \times U_{S}\right)$, and thus $\delta$-closed in $(X \times Y, T \times U)$.

\section{REFERENCES}

[B1] BAKER, C.W. Properties of subweakly continuous functions, Yokohama Math. J. 32(1984), 39-43.

[B2] BAKER, C.W. Characterizations of some near-continuous functions and near-open functions, Internat. J. Math. \& Math. Sci. $\underline{9}(1986), 715-720$.

[CN] CAMMAROTO, F. and NOIRI, T. On weakly $\theta$-continuous functions, Mat. Vesnik $\underline{38}(1986), 33-44$ 
[EJ] ESPELIE, M.S. and JOSEPH, J.E. Two weak forms of continuity, Canad. Math. Bu11. $25(1982), 59-63$.

[F] FOMIN, S. Extensions of topological spaces, Ann. of Math. $44(1943), 471-480$.

[GMRV] GAULD, D.B., MRŠEVIÉ, M., REILLY, I.L. and VAMANAMURTHY, M.K. Continuity properties of functions, Colloquia Math. Soc. Janos Bolyai 41 (1983), $311-322$.

[L] LEVINE, N. A decomposition of continuity in topological spaces, Amer. Math. Monthly $\underline{68}(1961), 44-46$.

[MRV] MRŠEVIC, M., REILLY, I.L. and VAMANAMURTHY, M.K. On semiregularization topologies, J. Austral. Math. Soc. Series A 38(1985), 40-54.

[N1] NOIRI, T. Between continuity and weak continuity, Bo11. U.M.I. 9(1974), 647-654.

[N2] NOIRI, T. Weak1y $\alpha$-continuous functions, Internat. J. Math. \& Math. Sci. $\underline{10}(1987), 483-490$.

[R] ROSE, D.A. Weak continuity and strongly closed sets, Internat. J. Math. E Math. Sci. $7(1984), 809-816$. 


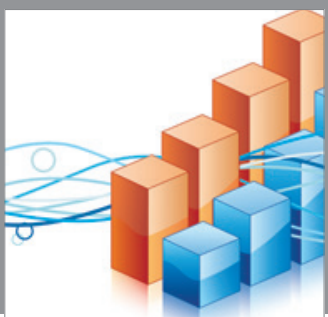

Advances in

Operations Research

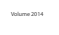

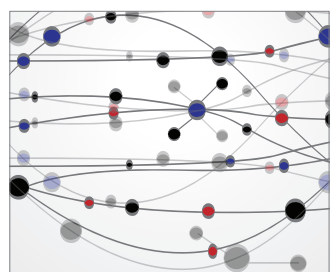

\section{The Scientific} World Journal
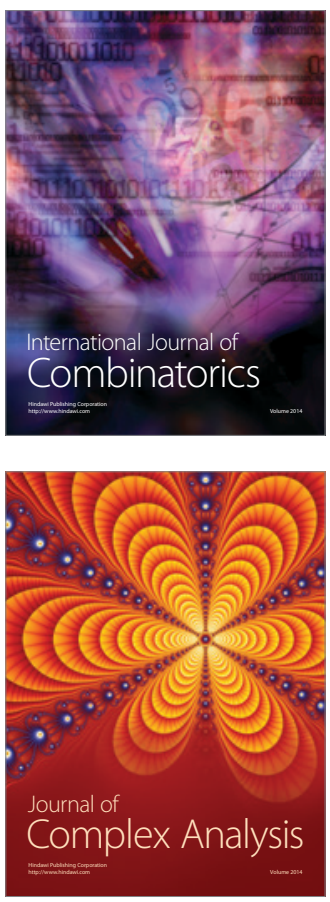

International Journal of

Mathematics and

Mathematical

Sciences
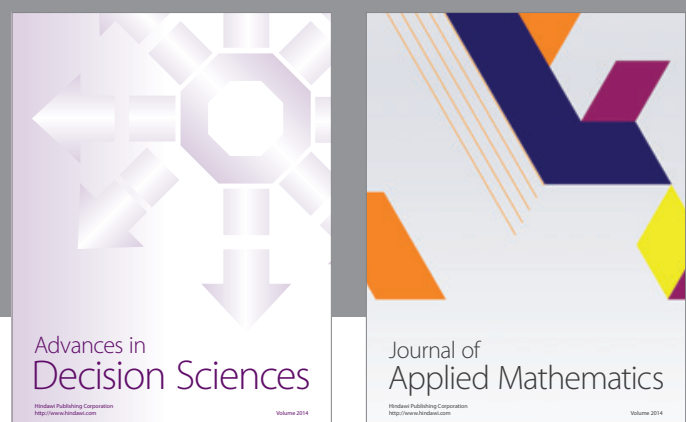

Journal of

Applied Mathematics
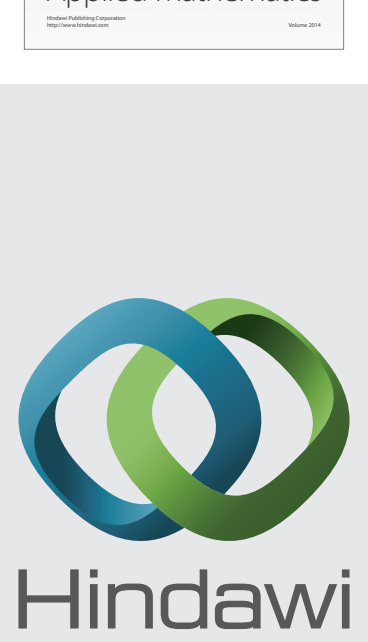

Submit your manuscripts at http://www.hindawi.com
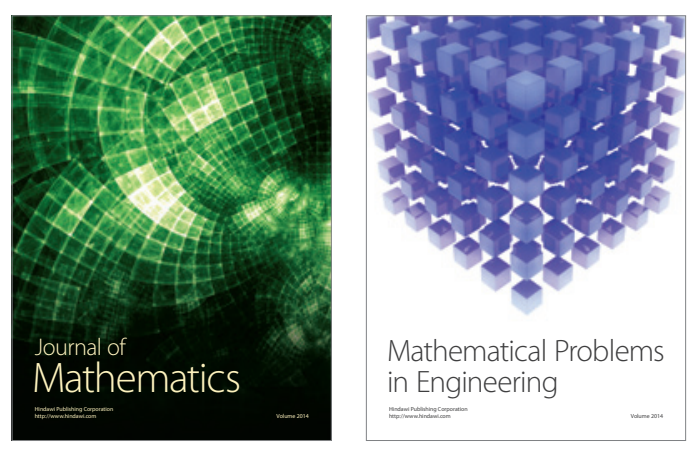

Mathematical Problems in Engineering
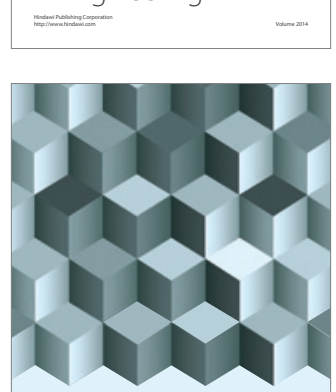

Journal of

Function Spaces
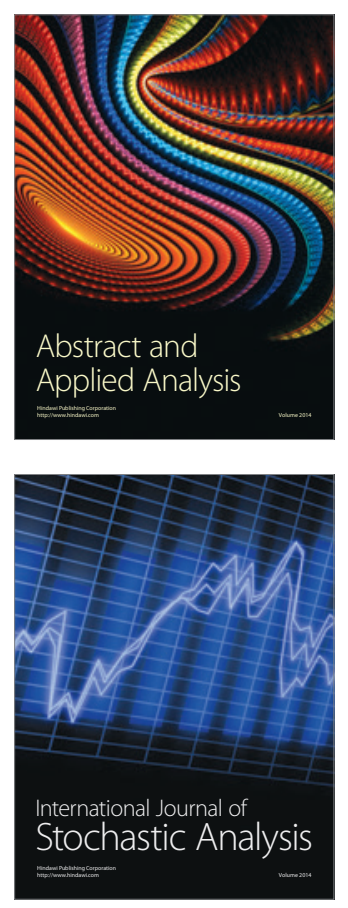

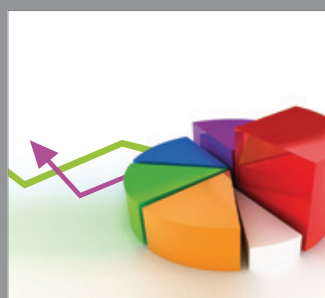

ournal of

Probability and Statistics

Promensencen
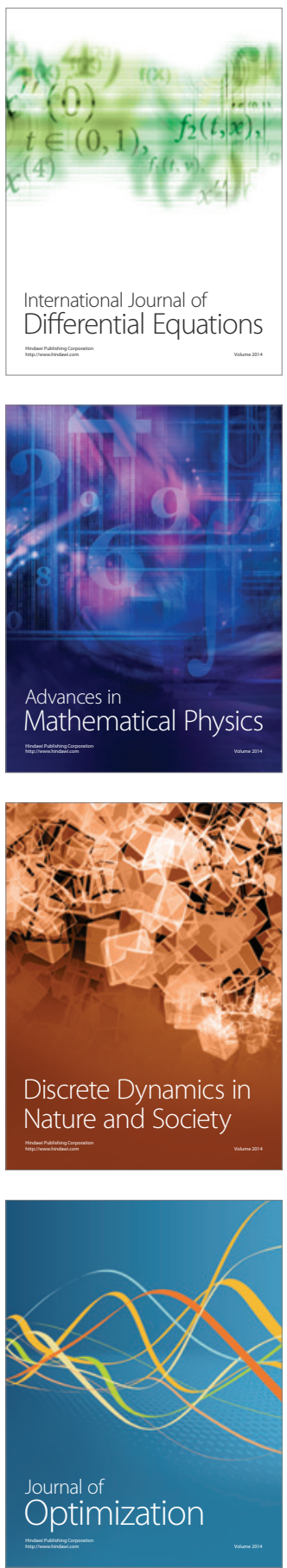